\title{
Dynamic Secondary Ion Mass Spectrometry (SIMS) Imaging of Materials for the Nuclear Industry: Historical Perspectives and Recent Advances
}

\author{
G. McMahon ${ }^{1}$, B.D. Miller ${ }^{2}$ and M.G. Burke ${ }^{3}$ \\ 1. University of Manchester, School of Materials, Manchester UK \\ 2. Naval Nuclear Laboratory, West Mifflin PA, USA \\ ${ }^{3}$ University of Manchester, Materials Performance Centre, Manchester UK
}

Dynamic SIMS is a microanalytical technique based on mass spectrometry analysis of sputtered sample ions resulting from a high dose bombardment of the specimen by an energetic beam of ions. The methodology and instrumentation was pioneered by Castaing and Slodzian [1] more than fifty years ago and modern instrumentation still shares many of the characteristics and design of that first instrument.

When we discuss dynamic SIMS imaging, it is important to distinguish between the two different manners in which SIMS images can be acquired. The first is by ion microscopy, sometimes also referred to as "ion microscope mode" where the location of a sputtered ion from the sample surface is preserved by the design of the ion optics in the final mass resolved image. In this sense, this mode of acquisition is analogous to optical microscopy where the resolution is dependent upon the optics of the microscope. Spatial resolution of mass resolved ion images acquired in this mode are limited to approximately $1 \mu \mathrm{m}$. Images may also be acquired in "ion microprobe" mode, which is exactly analogous to scanning electron microscopy where the spatial resolution is dependent upon the probe diameter. This allows for submicron spatial resolution. These instruments were commercialized by Cameca and collectively referred to as the "f-Series" of instruments.

In 1992 Slodzian et al [2] described a new scanning ion instrument with radically redesigned ion optics employing a coaxial lens, a system where a set of lenses are used to simultaneous shape and focus the primary ion beam and collect the secondary ion beam along the same optical axis. This enabled implementation of a much shorter working distance, reducing both primary beam aberrations and, because of the extremely high electric potential at the sample surface, the effect of large angular and energy dispersions of the secondary ion beam. This instrument evolved into the Cameca NanoSIMS.

A historical example of the application of the ion microscope mode of imaging to materials pertinent to the nuclear industry was the Birks Award winning presentation at this conference in 1999 [3]. In this example, ion microscopy was used to evaluate the resistance of carbide band dissolution in Alloy 600 subjected to different solution annealing protocols: As received, solution annealed at $1100^{\circ} \mathrm{C}$ for 4 hours followed by a water quench, and solution annealed at $1100^{\circ} \mathrm{C}$ for 4 hours followed by a furnace cool. Figure 1 shows a series of direct ion images for oxygen, boron and carbon. In the as-received condition, boron and carbon are present in micron sized carbides. Following the water quench, precipitates in the banded regions were observed to be rich in both boron and carbon, and as well boron segregation to grain boundaries outside of the banded region in the recrystallized matrix is also observed. The furnace cooled specimen indicated a higher density of precipitates enriched in both carbon and boron in the banded regions and segregation of both carbon and boron to some grain boundaries in the recrystallized matrix.

A recent example of how NanoSIMS imaging has been applied is in an investigation of the distribution 
of hydrogen ahead and in the wake of fatigue crack tips and at the interface of $\mathrm{MnS}$ inclusions in type 316 stainless steel fatigue tested at high stress ratio $(0.7)$, low delta $\mathrm{K}(\sim 11 \mathrm{MPa} \vee \mathrm{m})$, and a frequency of 1 cycle per minute in a high pressure $(68.9 \mathrm{MPa})$ gaseous deuterium $(99.999 \%$ purity) environment at a temperature of $22^{\circ} \mathrm{C}$ [4]. Figure 2a shows a Deuterium/Oxygen ratio with a $4 \mu \mathrm{m}$ field of view displayed as a hue-saturation intensity (HSI) image, where the hue represents the ratio value and the saturation represents the counting statistics (areas with poorer counting statistics are darker) while Figure 2b illustrates locations of ROI's generated by a segmentation algorithm showing contiguous regions with a minimum area of 50 pixels $^{2}$ and a mean Deuterium/Oxygen ratio at least $20 \%$ higher than the background taken from an area away from the crack tip. Figure 3 exhibits an example of the trapping of hydrogen at a MnS inclusion in the form of a ${ }^{32} \mathrm{~S}^{2} \mathrm{H} /{ }^{32} \mathrm{~S}$ HSI ratio image. The area of high ratio values indicated by the pink region surrounding the inclusion clearly demonstrates the trapping of hydrogen at the inclusion.

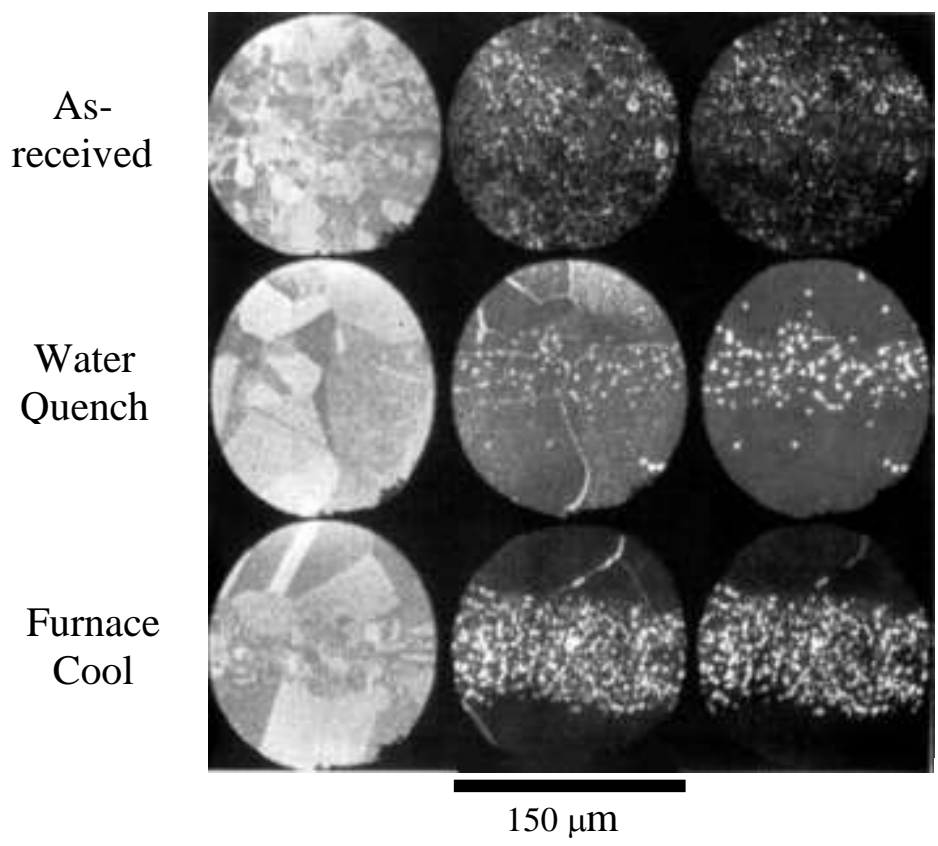

Figure 1: Direct ion images of oxygen (left), boron (middle) and carbon (right) from allow 600 in the asreceived condition and following solutions anneals at 1100C and either water quenched or furnace cooled. Diameter of images is $150 \mu \mathrm{m}$.

References:
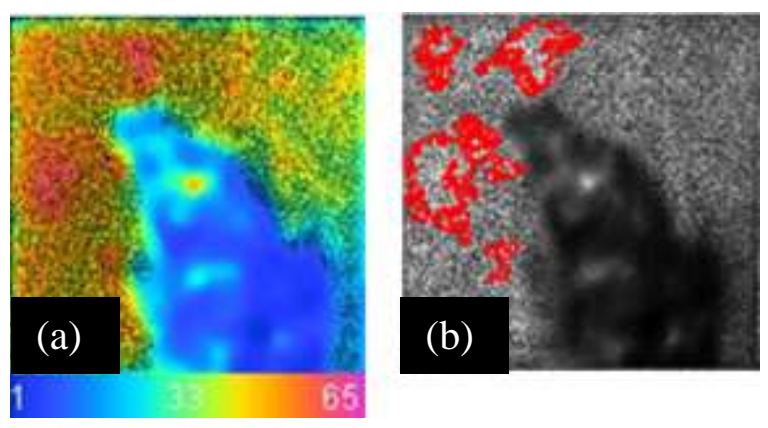

Figure 2: (a) Deuterium/Oxygen image shown as an HSI image and (b) Location of contiguous regions of interest generated by a segmentation algorithm with ratio values $20 \%$ higher than background value and at least 50 pixels $^{2}$ in size. Field of view is $4 \mu \mathrm{m}$.

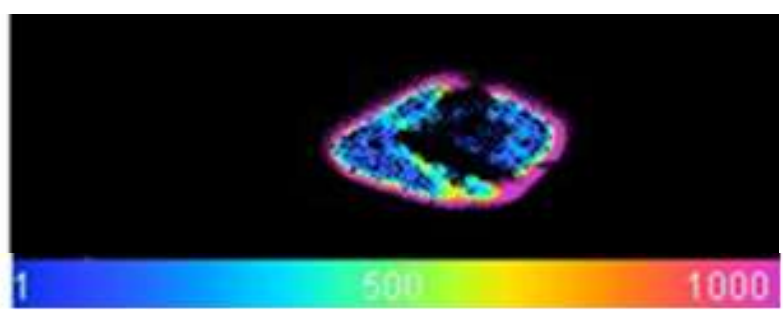

Figure 3: ${ }^{32} \mathrm{~S}^{2} \mathrm{H} /{ }^{32} \mathrm{~S}$ ratio as an HSI image. The area of high ratio values indicated by the pink region surrounding the inclusion demonstrates the trapping of hydrogen at the inclusion.

1. R. Castaing, G. Slodzian, J Microsc., 1(1962) p31-38.

2. G. Slodzian et al,. Biol Cell., 74 (1992) p43-50.

3. M.G. Burke, B.Z. Hyatt and G. McMahon, Microscopy and Microanalysis, 5 (1999) p862-863.

4. G. McMahon, B.D. Miller and M.G. Burke, submitted (2017). 